\title{
Serum Soluble TACI, a BLyS Receptor, Is a Powerful Prognostic Marker of Outcome in Chronic Lymphocytic Leukemia
}

\author{
Marie-Christine Kyrtsonis, ${ }^{1}$ Katerina Sarris, ${ }^{1}$ Efstathios Koulieris, ${ }^{1}$ \\ Dimitrios Maltezas, ${ }^{1}$ Eftychia Nikolaou, ${ }^{1}$ Maria K. Angelopoulou, ${ }^{1}$ \\ Vassiliki Bartzis, ${ }^{1}$ Tatiana Tzenou, ${ }^{1}$ Maria Dimou, ${ }^{1}$ Mariana P. Siakandaris, ${ }^{1}$ \\ Nora A. Viniou, ${ }^{1}$ Sotirios Sachanas, ${ }^{2}$ Christina Kalpadakis, ${ }^{2}$ Petros P. Sfikakis, ${ }^{1}$ \\ Gerassimos A. Pangalis, ${ }^{2}$ and Panayiotis Panayiotidis ${ }^{1}$ \\ ${ }^{1}$ Hematology Section of the First Department of Propedeutic Internal Medicine, Laikon University Hospital, \\ Agiou Thoma 17, 11527 Athens, Greece \\ ${ }^{2}$ Hematology Clinic of Athens' Medical Center, Andersen 1, Psychiko, 11525 Athens, Greece
}

Correspondence should be addressed to Marie-Christine Kyrtsonis; mck@ath.forthnet.gr

Received 17 April 2014; Accepted 20 July 2014; Published 6 August 2014

Academic Editor: Kazuyuki Shimizu

Copyright (C) 2014 Marie-Christine Kyrtsonis et al. This is an open access article distributed under the Creative Commons Attribution License, which permits unrestricted use, distribution, and reproduction in any medium, provided the original work is properly cited.

\begin{abstract}
BLyS is involved in CLL biology and its low soluble serum levels related to a shorter time to first treatment (TFT). TACI is a BLyS receptor and can be shed from cells' surface and circulate in soluble form (sTACI). We investigated the impact of serum BLyS and sTACI levels at diagnosis in CLL patients and their relationship with disease parameters and patients' outcome. Serum BLyS was determined in 73 patients, while sTACI in 60. Frozen sera drawn at diagnosis were tested by ELISA. sTACI concentrations correlated with BLyS $(P=-0.000021)$, b2-microglobulin $(P=0.005)$, anemia $(P=-0.03)$, thrombocytopenia $(P=0.04)$, Binet stage $(P=0.02)$, and free light chains ratio $(P=0.0003)$. Soluble BLyS levels below median and sTACI values above median were related to shorter TFT $(P=0.0003$ and 0.007$)$. During a ten-year followup, sTACI levels, but not BLyS, correlated with survival $(P=0.048)$. In conclusion, we confirmed the prognostic significance of soluble BLyS levels with regard to TFT in CLL patients, and, more importantly, we showed for the first time that sTACI is a powerful prognostic marker, related to parameters of disease activity and staging and, more importantly, to TFT and OS.
\end{abstract}

\section{Introduction}

Chronic lymphocytic leukemia (CLL) is an indolent leukemic B-cell lymphoproliferative disorder, defined in the WHO classification of neoplasms as a neoplasm of mature B-lymphocytes involving peripheral blood, bone marrow, spleen, and lymph nodes [1]. It is the most common form of leukemia in the elderly in the Western world. For diagnosis a minimum of $5 \times 10^{9} / \mathrm{L}$ absolute blood lymphocyte count is required; these typically coexpress CD5, CD19, CD20, and CD23 antigens with dim CD20, CD79b, and surface immunoglobulin expression [2,3]. Patients usually enjoy a relatively favourable outcome and the majority of them are asymptomatic and may not need any treatment for years. However, some patients have a more aggressive course and shorter survival; clinical manifestations, when present, include anaemia, peripheral lymphadenopathy, splenomegaly, and autoimmune manifestations. Treatment should be immediately started in presence of a lymphocyte doubling time of less than 6 months, very enlarged or rapidly growing lymph nodes or spleen, anemia, thrombocytopenia, and B-symptoms. For symptomatic patients, adverse prognostic factors include classical staging (according to Rai or Binet), unmutated VH genes, ZAP-70 and CD38 expression, and cytogenetic alterations such as deletion of 11q22, deletion of 17p, and/or presence of a TP53 mutation [4]. Additional prognostic factors are needed for asymptomatic patients to predict whether they will remain stable for years or not. 
TABle 1: Patients' characteristics at the time of diagnosis.

\begin{tabular}{|c|c|c|}
\hline \multirow[t]{2}{*}{ Age, median (range) } & \multicolumn{2}{|c|}{60 years $(37-82)$} \\
\hline & For BLyS measurements & For sTACI measurements \\
\hline \multirow[t]{2}{*}{$N$} & 73 & 60 \\
\hline & \multicolumn{2}{|c|}{$\%$} \\
\hline Sex, M/F & $61 / 39 \%$ & $66 / 34 \%$ \\
\hline \multicolumn{3}{|l|}{ Binet stage } \\
\hline 1 & $59 \%$ & $60 \%$ \\
\hline 2 & $34 \%$ & $32 \%$ \\
\hline 3 & $7 \%$ & $8 \%$ \\
\hline Lymphadenopathy & $60 \%$ & $60 \%$ \\
\hline Splenomegaly & $18 \%$ & $15 \%$ \\
\hline Haemoglobin $<10 \mathrm{~g} / \mathrm{dL}$ & $5.5 \%$ & $8.3 \%$ \\
\hline Platelet counts $<100 \times 10^{9} / \mathrm{L}$ & $2.7 \%$ & $3.3 \%$ \\
\hline Abnormal LDH & $15 \%$ & $15 \%$ \\
\hline BM infiltration $>50 \%$ & $59 \%$ & $50 \%$ \\
\hline$\beta 2$-Microglobulin $>3.5 \mathrm{mg} / \mathrm{L}$ & $30 \%(12 / 39$ pts $)$ & $27 \%$ (9/33 pts) \\
\hline Abnormal $\kappa$-sFLC (normal 3.3-19.4 mg/L) & $30 \%(20 / 67 \mathrm{pts})$ & $30 \%(17 / 56 \mathrm{pts})$ \\
\hline Abnormal $\lambda$-sFLC (normal 5.71-26.3 mg/L) & $8 \%(3 / 67 \mathrm{pts})$ & $5 \%(3 / 56 \mathrm{pts})$ \\
\hline
\end{tabular}

FLC: free light chain.

B-lymphocyte stimulator (BLyS) is a cytokine, member of the TNF-superfamily, that is involved in CLL biology and was shown to regulate B-CLL cells proliferation and survival [5]. Furthermore, serum BLyS levels were found decreased in CLL patients and their low concentrations related to a shorter time to first treatment (TFT) but not to overall survival (OS) [6]. BLyS is produced by myeloid cells, monocytes, dendritic cells, and osteoclasts [7]. It may be cleaved from cells' surface and circulate in body fluids in a soluble form [8]. BLyS actions concern almost exclusively cells of lymphoid lineage and are exerted through its receptors [9]. TACI (transmembrane activator and CAML interactor) is one of the 3 BLyS (BAFF) receptors and is expressed by $\mathrm{B}$ and $\mathrm{T}$ cells. It can also bind APRIL. TACI can also be shed from cells' surface and circulate in its soluble form $[10,11]$. Very few studies so far investigated soluble TACI (sTACI) serum levels in CLL.

The purpose of this study was to investigate possible relationship between serum BLyS and sTACI concentrations at diagnosis in CLL, as well as eventual correlations of their respective levels with disease parameters and patients' outcome.

\section{Patients and Methods}

Seventy-three CLL patients were studied. Their characteristics are shown in Table 1.

Sera from patients were drawn at diagnosis and aliquots were kept frozen and retrospectively tested to determine BLyS and sTACI concentrations. Frozen sera from 14 healthy individuals (HI) for BLyS and sTACI, respectively, were also tested as controls.

42 patients were or became symptomatic and in need of treatment during disease course. Patients' median OS was 79 months (range 18-174) while median TFT was 34 months (range 1-157).
Serum BLyS levels were determined by ELISA (R\&D Quantikine KIT) in all 73 patients while sTACI (R\&D Quantikine, DuoSet) in 60 of them, according to the manufacturer's instruction; briefly (1) for BLys determination, the specified amount of patients' or HI serum was added to a 96cell microplate already coated with a capture antibody. After incubation the plate was washed, the detection antibody was added, and after another incubation and wash StreptavidinHRP and Tetramethylbenzidine in $\mathrm{H}_{2} \mathrm{O}_{2}$ were added, and finally $\mathrm{H}_{2} \mathrm{SO}_{4}$ was added to stop the reaction. (2) For sTACI determination, a 96-cell microplate was coated with the specified amount of capture antibody and, after being incubated overnight and washed, Reagent Diluent was added to block the plate and after another wash the specified amount of patients' or controls' sera was added. The subsequent procedure was almost identical (washing, addition of detection antibody, incubation, washing, and addition of StreptavidinHRP followed by Tetramethylbenzidine in $\mathrm{H}_{2} \mathrm{O}_{2}$ solution and then stop solution). In order to determine the values of both BLys and sTACI, the optical density of each cell was determined at $450+620 \mathrm{~nm}$ by using a photometer. On a biaxial system where $x$-axis was the serum concentration of the cytokine and $y$ was optical density, the sera cytokine concentration was determined using a curve plotted based on the optical density of known serial concentrations.

Statistical analysis was performed using the SPSS v.15 software. Nonparametric variables were compared by the Mann-Whitney test. TFT and OS curves according to BLyS or sTACI levels were assessed and plotted by the Kaplan-Meier analysis and then compared by the log-rank test. $P$ values of less than 0.05 were considered statistically significant.

\section{Results and Discussion}

3.1. Serum BLyS and sTACI Levels. In the present study, median serum BLyS levels were $65 \mathrm{pg} / \mathrm{mL}$ (undetectable -680) while 


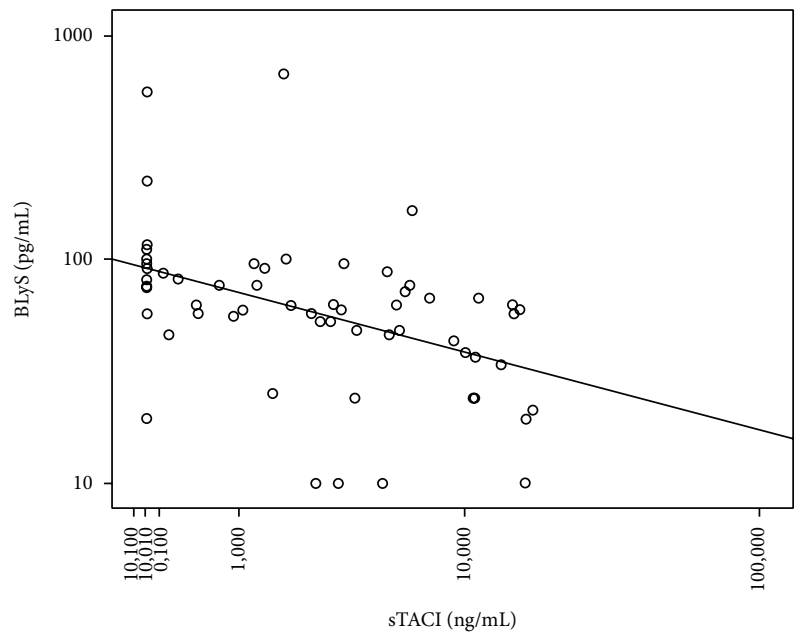

FIGURE 1: Inverse correlation between serum soluble BLyS and sTACI.

sTACI's ones were 2,52 ng/mL (undetectable -17) in CLL patients. The corresponding median concentrations in $\mathrm{HI}$ sera samples were $183 \mathrm{pg} / \mathrm{mL}$ (undetectable -381) and $0.86 \mathrm{ng} / \mathrm{mL}$ (undetectable $-4,14$ ) for BLyS and sTACI, respectively. The difference between levels in patients and $\mathrm{HI}$ is significant for both $(P<0.01)$. In addition, sTACI concentrations strongly correlated inversely with soluble BLyS $(P=$ 0.000021), as shown in Figure 1.

In accordance with our results, serum BLyS levels were reported lower in CLL patients as compared to HI, in several previous studies [12-14]. However, with regard to sTACI levels in CLL, that we found higher in patients than in HI, there are no reports in medical literature, so far, to our knowledge.

As an attempt to explain the low serum BLyS levels found in CLL patients, Molica et al. [14] suggested that in aggressive CLL high amounts of soluble BLyS are bound by its receptors on B-cell surface, thus sequestrating it from circulation. This theory was reinforced by another study that showed that serum BLyS concentrations increased in follicular lymphoma patients after rituximab administration, possibly because it is unbounded by its surface receptors [15]. In view of our finding of a strong inverse correlation between BLyS and sTACI serum concentrations, one could assume that binding of soluble BLyS to sTACI results in hiding the BLyS epitope that is detected by ELISA measurements and that BLyS levels are falsely low. This supposition remains indeed to be proved but if it was the case, it would have significant therapeutical implications, given that anti-BLyS or anti-BLyS receptors antibodies have been manufactured for adjuvant treatment in B-cell lymphoproliferative disorders $[16,17]$ but are not considered for CLL for which caution is needed until BLyS contribution to disease biology is fully understood.

3.2. Correlations between BLyS or sTACI Levels and Disease Parameters. Serum BLyS levels correlated inversely with absolute lymphocyte count $(P=0.01$, Spearman's rho $=$ $-0.303)$. Furthermore an inverse correlation was demonstrated for BLyS values above median and bone marrow

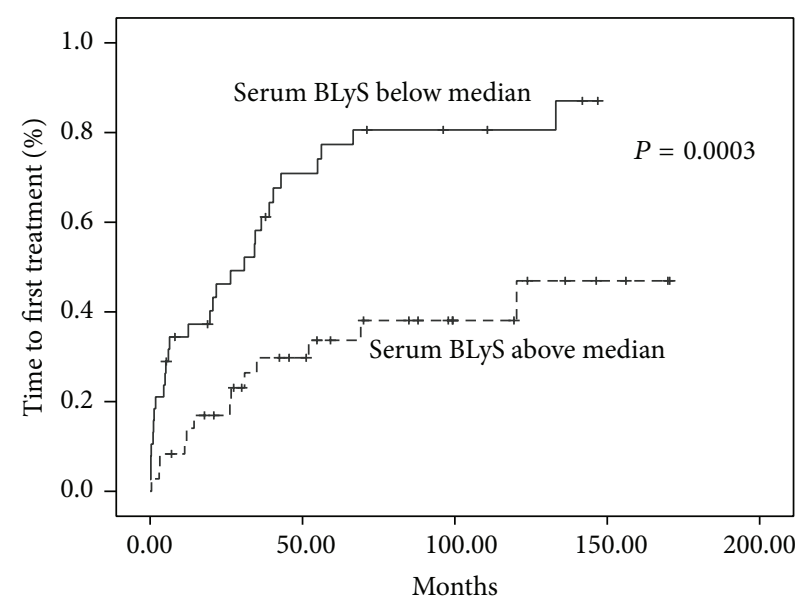

FIgURE 2: Time to first treatment according to serum soluble BLyS.

lymphocyte infiltration greater than 50\% $(P=0.031$, chi square) while sTACI concentrations correlated with b2microglobulin $(P=0.005)$, inversely with anemia $(P=0.03)$, thrombocytopenia $(P=0.04)$, Binet stage $(P=0.02)$, and free light chains ratio $(P=0.0003)$. In Molica et al's study higher BLyS levels were associated with younger age, higher platelet count, mutated $\mathrm{IgVH}$, normal cytogenetic profile or presence of $13 \mathrm{q}$ deletion, and low ZAP-70 and CD38 expression. Unfortunately, in our series mutational status and cytogenetic abnormalities were determined in only $15 \%$ of patients rendering statistical analysis impossible; ZAP-70 expression was not determined and there was no correlation with CD38 expression. With regard to sTACI levels, given that this is the first report, we cannot compare with other studies.

3.3. Time to First Treatment and Overall Survival according to BLyS and sTACI Serum Concentrations. In CLL patients serum BLyS values below median were related to a significantly shorter TFT compared to values above median $(P=$ 0.0003) (Figure 2) but no correlation was found with overall survival. In addition, serum sTACI values above median were also related to a shorter TFT compared to values below median $(P=0.007)$ and, importantly, also to OS $(P=0.048$, HR: 2.789, 95\% CI: 0.967-8.047), as shown in Figure 3. Serum sTACI values above median maintained their negative prognostic impact in the Cox Regression model when tested with known disease parameters such as Rai and Binet stadium, b2microglobulin, and LDH.

Lower soluble BLyS levels have already been associated with a shorter time to first treatment $[14,18]$ but not with overall survival, most probably because of the indolent nature of the disease, requiring a very long follow-up time, in order to show survival advantage. Of special interest are our findings concerning the prognostic significance of sTACI with regard to both TFT and OS; such findings have not been reported so far in medical literature. Because the vast majority of our patients were not in need of treatment and low staged at diagnosis, our results suggest that serum sTACI could be a new, easily assessed, marker for predicting CLL behaviour in low staged patients. Such prognostic factors are missing for 


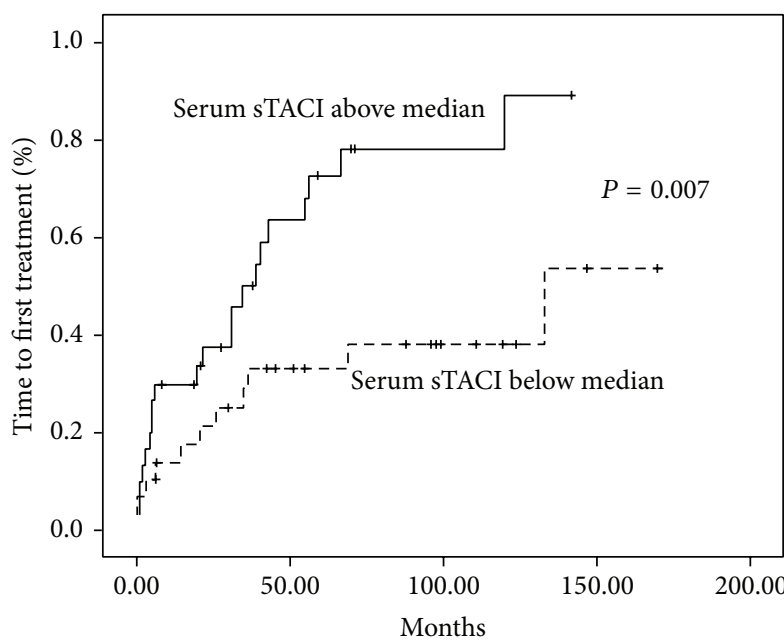

(a)

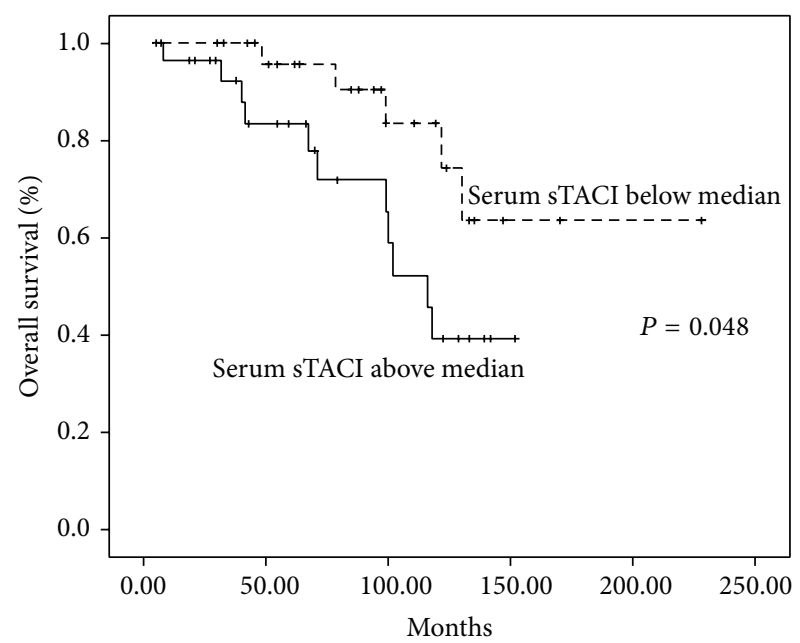

(b)

FIGURE 3: Time to first treatment (a) and overall survival (b) according to serum sTACI.

asymptomatic patients although in this context serum free light chain measurements appear very promising [19-21].

\section{Conclusions}

We confirmed that low soluble BLyS are associated with a shorter time to first treatment while in addition we found that sTACI serum concentrations at diagnosis constitute a powerful prognostic marker in chronic lymphocytic leukemia; sTACI is related to disease activity parameters and the stage of CLL and more importantly, sTACI levels above median predicted a shorter time to first treatment and worse outcome for the patients. Indeed these results are preliminary and concern a relatively short series, although with a very long follow-up, and further researches are needed. However, if confirmed, our results suggest that sTACI could be a valuable prognostic marker in CLL while, in addition, they could open interesting therapeutical applications.

\section{Ethical Approval}

The study was approved by the local ethical committee.

\section{Conflict of Interests}

The authors declare that there is no conflict of interests regarding the publication of this paper.

\section{References}

[1] S. H. Swerdlow, E. Campo, and N. E. Harris, Eds., WHO Classification of Tumours of Haematipoetic and Lymphoid Tissues, Lyon IARC Press, 2008.

[2] M. Hallek, B. D. Cheson, D. Catovsky et al., "Guidelines for the diagnosis and treatment of chronic lymphocytic leukemia: a report from the International Workshop on Chronic Lymphocytic Leukemia updating the National Cancer InstituteWorking Group 1996 guidelines," Blood, vol. 111, no. 12, pp. 5446-5456, 2008.
[3] E. Matutes, K. Owusu-Ankomah, R. Morilla et al., "The immunological profile of B-cell disorders and proposal of a scoring system for the diagnosis of CLL," Leukemia, vol. 8, no. 10, pp. 1640-1645, 1994.

[4] P. Cramer and M. Hallek, "Prognostic factors in chronic lymphocytic leukemia - what do we need to know?" Nature Reviews Clinical Oncology, vol. 8, no. 1, pp. 38-47, 2011.

[5] A. J. Novak, R. J. Bram, N. E. Kay, and D. F. Jelinek, "Aberrant expression of B-lymphocyte stimulator by B chronic lymphocytic leukemia cells: a mechanism for survival," Blood, vol. 100, no. 8, pp. 2973-2979, 2002.

[6] M.-C. Kyrtsonis, K. Sarris, T. Tzenou, and D. Maltezas, "Blymphocyte stimylator (BLyS) role in chronic lymphocytic leukaemia (CLL), Waldenström's Macroglobulinaemia (WM) and Multiple Myeloma (MM). Treatment strategies," Hematology, 2013.

[7] A. Craxton, D. Magaletti, E. J. Ryan, and E. A. Clark, "Macrophage- and dendritic cell-dependent regulation of human B-cell proliferation requires the TNF family ligand BAFF, Blood, vol. 101, no. 11, pp. 4464-4471, 2003.

[8] F. Melchers, "Actions of BAFF in B cell maturation and its effects on the development of autoimmune disease," Annals of the Rheumatic Diseases, vol. 62, supplement 2, pp. ii25-ii27, 2003.

[9] F. Mackay and C. Ambrose, "The TNF family members BAFF and APRIL: the growing complexity," Cytokine and Growth Factor Reviews, vol. 14, no. 3-4, pp. 311-324, 2003.

[10] F. Mackay and J. L. Browning, "BAFF: a fundamental survival factor for B cells," Nature Reviews Immunology, vol. 2, no. 7, pp. 465-475, 2002.

[11] F. Mackay, P. Schneider, P. Rennert, and J. Browning, "BAFF and APRIL: a tutorial on B cell survival," Annual Review of Immunology, vol. 21, pp. 231-264, 2003.

[12] G. Ferrer, K. Hodgson, E. Montserrat, and C. Moreno, "B cell activator factor and a proliferation-inducing ligand at the crossroad of chronic lymphocytic leukemia and autoimmunity," Leukemia and Lymphoma, vol. 50, no. 7, pp. 1075-1082, 2009. 
[13] L. Planelles, S. Castillo-Gutiérrez, J. P. Medema, A. MoralesLuque, H. Merle-Béral, and M. Hahne, "APRIL but not BLyS serum levels are increased in chronic lymphocytic leukemia: prognostic relevance of APRIL for survival," Haematologica, vol. 92, no. 9, pp. 1284-1285, 2007.

[14] S. Molica, G. Digiesi, C. Battaglia et al., "Baff serum level predicts time to first treatment in early chronic lymphocytic leukaemia," European Journal of Haematology, vol. 85, no. 4, pp. 314-320, 2010.

[15] S. M. Ansell, A. J. Novak, S. Ziesmer et al., "Serum BLyS levels increase after rituximab as initial therapy in patients with follicular grade 1 non-Hodgkin lymphoma," The American Journal of Hematology, vol. 84, no. 2, pp. 71-73, 2009.

[16] J.-F. Rossi, J. Moreaux, D. Hose et al., "Atacicept in relapsed/ refractory multiple myeloma or active Waldenström's macroglobulinemia: a phase I study," British Journal of Cancer, vol. 101, no. 7, pp. 1051-1058, 2009.

[17] N. S. Raje, R. J. Hohl, E. A. Faber et al., "Phase I study of LY2127399, a human anti-BAFF antibody, and bortezomib in patients with previously treated multiple myeloma," Journal of Clinical Oncology, vol. 29, supplement, abstract 8012, 2011.

[18] M. C. Kyrtsonis, K. Sarris, G. Pangalis et al., "Contradictory impact of serum BLyS levels in Chronic Lymphocytic leukemia, Waldestrom's macroglobulinemia and Multiple Myeloma," EHA, Abstr 741, 2012.

[19] G. Pratt, S. Harding, R. Holder et al., "Abnormal serum free light chain ratios are associated with poor survival and may reflect biological subgroups in patients with chronic lymphocytic leukaemia," The British Journal of Haematology, vol. 144, no. 2, pp. 217-222, 2009.

[20] F. Morabito, R. de Filippi, L. Laurenti et al., “The cumulative amount of serum-free light chain is a strong prognosticator in chronic lymphocytic leukemia," Blood, vol. 118, no. 24, pp. 63536361, 2011.

[21] K. Sarris, D. Maltezas, E. Koulieris et al., "Prognostic significance of serum free light chains in chronic lymphocytic leukemia," Advances in Hematology, vol. 2013, Article ID 359071, 7 pages, 2013. 


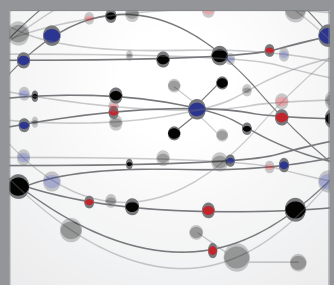

The Scientific World Journal
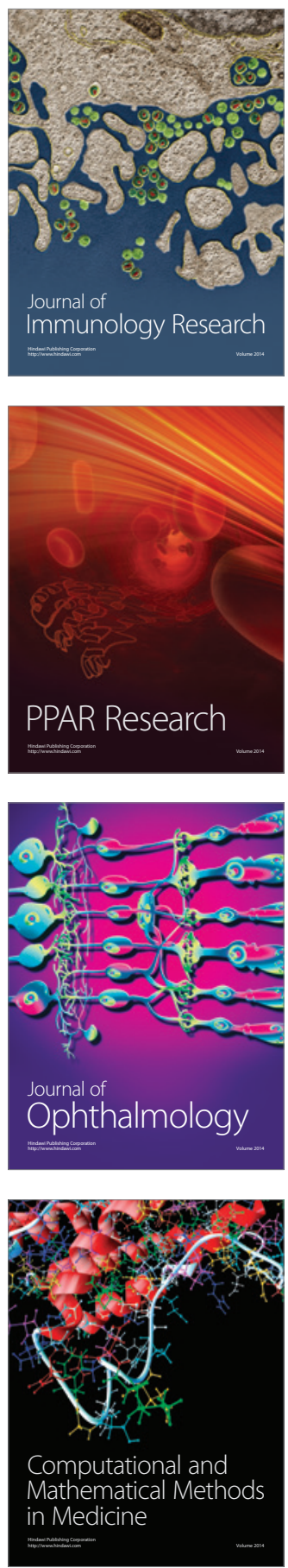

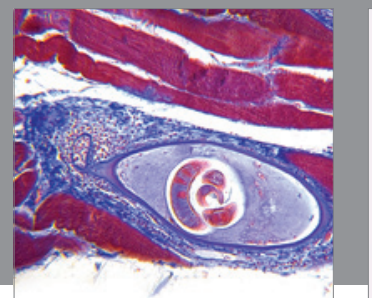

Gastroenterology

Research and Practice
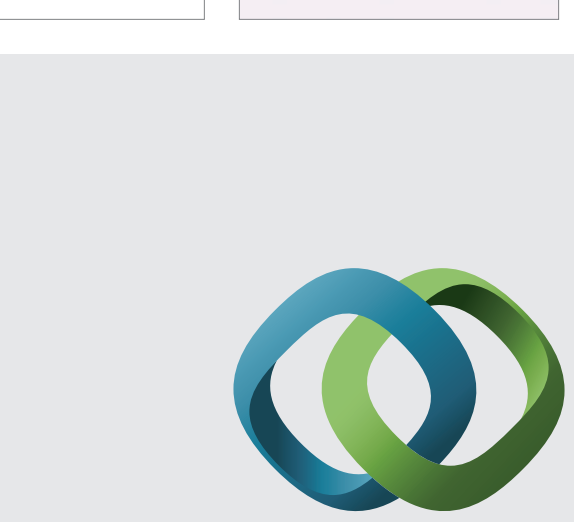

\section{Hindawi}

Submit your manuscripts at

http://www.hindawi.com
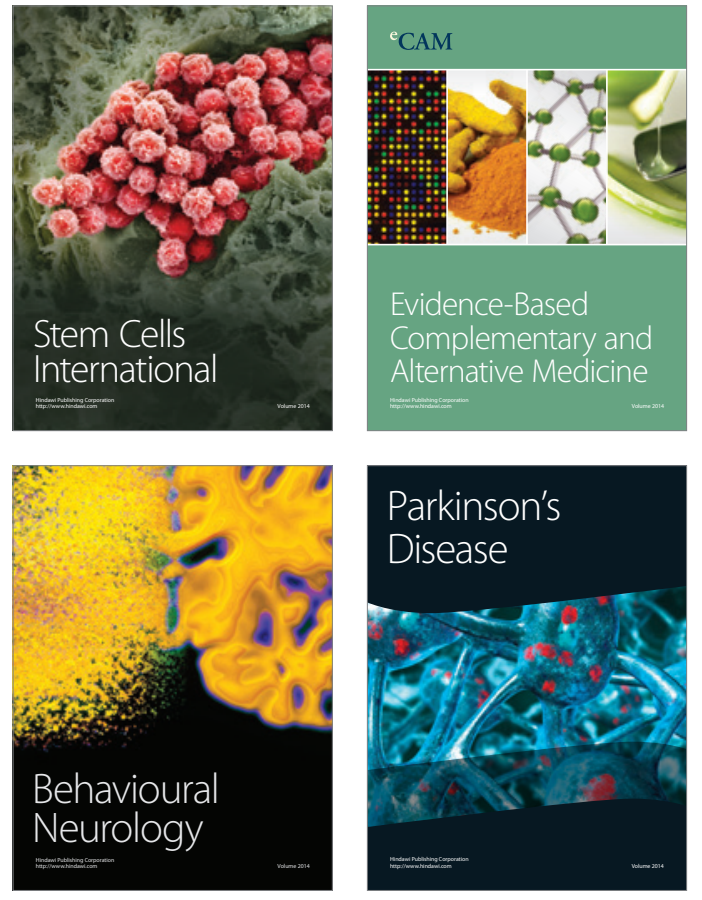
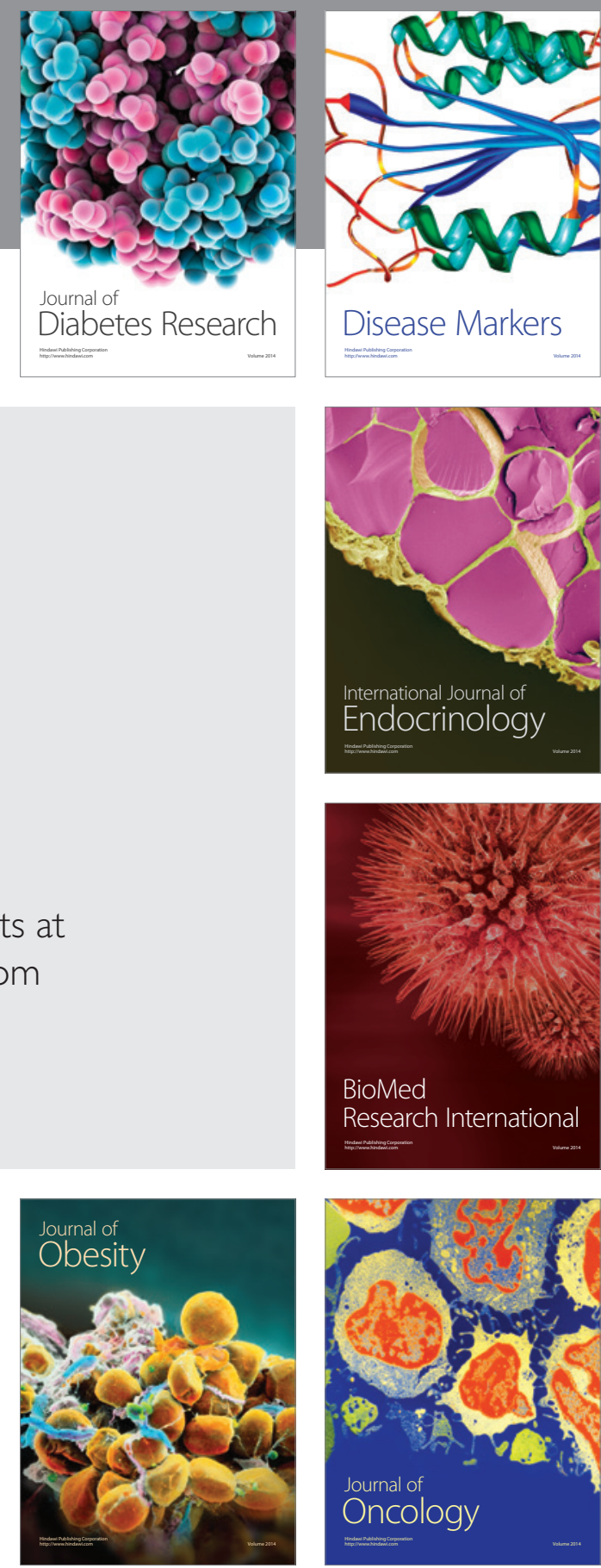

Disease Markers
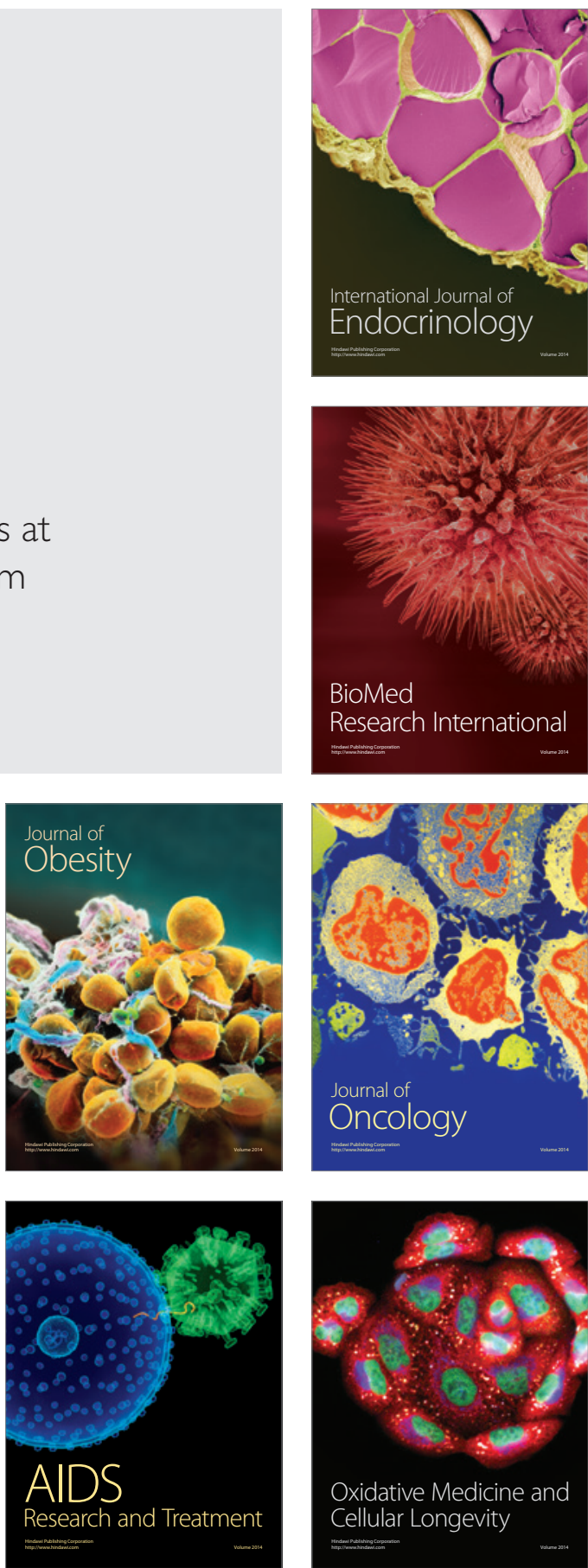